\title{
Contribution à une histoire de l'archéologie dans l'ouest de la France ou comment naît un laboratoire de recherche (1945-1980)
}

A story of archaeology in the west of France or how a research body was created (1945-1980)

Marie-Yvane Daire, Elías López-Romero, Jean-Laurent Monnier et Nathalie Richard

\section{OpenEdition \\ Journals}

Édition électronique

URL : http://journals.openedition.org/rao/5813

DOI : $10.4000 /$ rao.5813

ISSN : 1775-3732

Éditeur

Presses universitaires de Rennes

Édition imprimée

Date de publication : 18 décembre 2020

Pagination : 9-21

ISBN : 978-2-7535-8232-3

ISSN : 0767-709X

Référence électronique

Marie-Yvane Daire, Elías López-Romero, Jean-Laurent Monnier et Nathalie Richard, « Contribution à une histoire de l'archéologie dans l'ouest de la France ou comment naît un laboratoire de recherche (1945-1980) », Revue archéologique de l'Ouest [En ligne], 36 | 2020, mis en ligne le 18 décembre 2020, consulté le 03 avril 2021. URL : http://journals.openedition.org/rao/5813 ; DOI : https://doi.org/ $10.4000 /$ rao. 5813 


\title{
Contribution à une histoire de l'archéologie dans l'ouest de la France ou comment naît un laboratoire de recherche (1945-1980)
}

\author{
A story of archaeology in the west of France or how a research body was created \\ (1945-1980)
}

\author{
Marie-Yvane DAIre ${ }^{a}$, Elías López-Romero ${ }^{b}$, Jean-Laurent MonnieR ${ }^{a}$ \\ et Nathalie RicharD ${ }^{c}$
}

\begin{abstract}
Résumé : Cet article propose un panorama historiographique de la structuration de l'archéologie dans l'ouest de la France. Il vise à retracer l'histoire, toujours complexe et particulière, de la formation d'un laboratoire de recherche, et d'en analyser les étapes fondatrices jusqu'aux années 1980. Au-delà de l'évolution de la discipline dans le contexte national, ce sont des personnages et des événements qui influent toujours sur le cours de cette formation. Pour les phases les plus anciennes (fin $\mathrm{XIX}^{\mathrm{e}}$ et première moitié du XX⿳⺈冂大 s.), le fonds d'archives du Laboratoire ArchéosciencesCReAAH de l'université de Rennes 1 se révèle une source d'une valeur primordiale, dont l'exploitation est en cours depuis plusieurs années. Pour les périodes suivantes, marquées par la professionnalisation de la discipline, la conjugaison des témoignages et des souvenirs de certains acteurs avec la documentation disponible illustrera les différentes étapes de cette évolution. Cette contribution se veut également un témoignage offrant aux jeunes générations une mise en perspective sur la longue durée de la discipline archéologique et de ses institutions.
\end{abstract}

\begin{abstract}
This paper provides a historiographical overview of the structuring of archaeology in Western France. It aims to retrace the complex and distinctive history a research laboratory from its beginnings until the 1980's which was influenced by scholars and events within the framework of its development on a national level. The archives of the Archéosciences-CReAAH laboratory at University of Rennes 1 have been the subject of research and conservation for several years and has proved to be a source of paramount importance for the study of the earliest moments (end of the 19th and first half of the 20th century) of the discipline in the region. The following periods are marked by its professionalisation, with the testimonies of the people involved and the written and iconographical sources documenting its evolution. This paper also offers the wider perspective of a long-term approach to archaeology and its institutions for future generations.
\end{abstract}

Mots clés : historiographie, archéologie, préhistoire, archives.

Keywords: history of archaeology, archaeology, prehistory, archives.

\section{INTRODUCTION}

Cet article propose de retracer les grandes lignes de l'histoire, à la fois particulière et complexe, de la formation d'un laboratoire de recherche spécialisé en archéologie, lato sensu, regroupant aujourd'hui la plupart des forces vives du grand quart nord-ouest de la France. À travers cette démarche, il s'agit de porter un éclairage sur les orientations scientifiques de ce laboratoire, son évolution et son identité présente, tout en les resituant dans le contexte plus large du développement et de l'institutionnalisation de l'archéologie dans l'ouest de la France.

\footnotetext{
a UMR 6566, CReAAH Centre de Recherche en Archéologie, Archéosciences, Histoire, RENNES (marie-yvane.daire@univ-rennes1.fr) (jean-laurent. monnier@univ-rennes1.fr)

b Universidad Complutense, MADRID, membre associé au CReAAH (eliaslop@ucm.es)

c TEMOS (Temps, Mondes, Sociétés, CNRS UMR 9016), Le Mans Université (nathalie.richard@univ-lemans.fr)
} 
La photographie actuelle de l'Unité Mixte de Recherche "Centre de Recherche en Archéologie, Archéosciences, Histoire " (UMR 6566 CReAAH) révèle en 2020 une communauté de 170 chercheurs, ingénieurs et doctorants, aujourd'hui largement regroupés sur plusieurs sites majeurs (Rennes, Nantes et Le Mans) mais, dans le détail, disséminés dans un grand quart nord-ouest de la France, de Caen à Poitiers. Cette dissémination peut être sans doute rapportée à la densité et à l'ancienneté de la recherche archéologique sur cette partie du territoire national. Si bien que cette photographie du paysage de la recherche actuelle gagne à être éclairée par l'histoire de la formation et de l'évolution de cet écosystème, afin de comprendre les tenants du paysage actuel de la recherche interrégionale. Cette histoire éclaire non seulement les caractéristiques géographiques et institutionnelles de cette unité mais aussi les objectifs et la nature des thématiques de recherche qui y sont développées.

Sur le site rennais, l'actuel laboratoire basé à l'université de Rennes 1, hérité de l'ancien Laboratoire d'Anthropologie Préhistorique, fondé par Pierre-Roland Giot (1919-2002) au début des années 1950, a fusionné avec le Laboratoire d'Archéométrie dans les années 1990 et est aujourd'hui devenu le Laboratoire Archéosciences. Autour du noyau central historique de l'ancien "Laboratoire d'Anthropologie " de la faculté des sciences, le processus de constitution de l'UMR 6566 résulte d'un agrégat progressif d'entités basées à la faculté des lettres de Rennes et au sein des universités de Nantes et du Mans.

Notre propos est centré sur l'histoire du laboratoire depuis sa création, sans toutefois développer les trois dernières décennies. Il est cependant impossible de faire l'impasse sur l'éclairage des origines et donc des périodes antérieures puisqu'elles ont été déterminantes pour la suite de l'histoire de la recherche régionale.

\section{Le rôle central de Pierre-Roland Giot ET DU LABORATOIRE D'ANTHROPOLOGIE}

Pierre-Roland Giot (1919-2002) est le personnage central dans la recherche préhistorique et archéologique de la seconde moitié du $\mathrm{xx}^{\mathrm{e}}$ s., dans le Grand Ouest français. Nous ne reviendrons pas ici sur les travaux de P.-R. Giot, traités par ailleurs (Collectif, 1990, 2002a), mais insistons sur son rôle structurant, à long terme, dans le paysage de la recherche régionale, dans une démarche que l'on qualifie aujourd'hui d'interdisciplinaire, conciliant les approches scientifiques (géologie appliquée à l'archéologie mais aussi histoire des paysages) à une démarche diachronique où seule l'Antiquité demeura en dehors de ses centres d'intérêt. Formé à la Préhistoire sur le terrain par des archéolo- gues amateurs, P.-R. Giot ne se distingue pas sur ce point des préhistoriens des premières générations qui, depuis les années 1850 , animent la discipline hors de tout cadre universitaire. Mais il est contemporain de réformes qui engagent l'institutionnalisation académique du champ et sa professionnalisation, avec la création du Centre National de la Recherche Scientifique en 1939 et la loi réglementant les fouilles archéologiques de 1941. Son parcours est représentatif de ces évolutions et des restructurations qu'elles entraînent pour l'archéologie préhistorique, avec la concentration progressive de la recherche au sein des laboratoires universitaires, la relégation - tout aussi progressive - au second plan des amateurs et de leurs associations et la perte de dynamisme des musées archéologiques non rattachés à des structures académiques.

En 1937, alors qu'il est encore un jeune étudiant, Giot assiste à la dernière campagne de fouilles de Georges Boisselier sur le tertre néolithique de Parc-ar-C'hastel à Tréguennec (Finistère). À la faveur de ses vacances à La Forêt-Fouesnant (Finistère), il réalise l'inventaire des mégalithes des communes environnantes. Ses premiers travaux s'orientent pourtant vers la géologie dans le cadre d'un parcours universitaire qu'il effectue à Grenoble, en participant notamment à la création d'une carte géologique dans l'Isère et la Drôme, qui est publiée en 1943. Cette même année, il entre comme chercheur au Centre National de la Recherche Scientifique (CNRS) et décide de travailler en Bretagne, sur les briovériens du sud des Montagnes noires. À l'Institut de Géologie de la faculté des

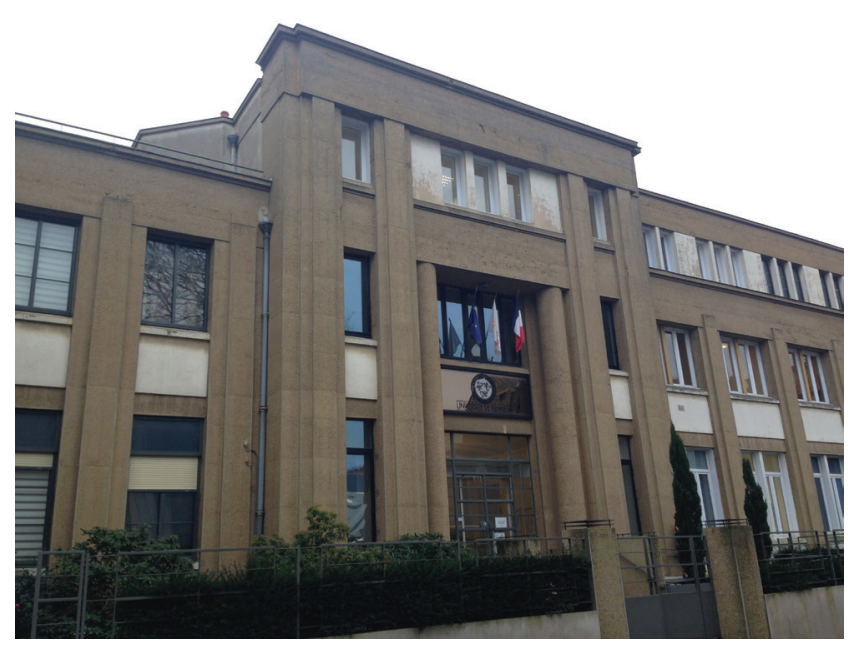

Figure 1 : Façade de l'ancien Institut de géologie, créé en 1937 par Y. Milon, aujourd'hui présidence de l'université Rennes 1 (cliché M.-Y. Daire, 2020).

Figure 1: Facade of the former Institute of Geology, created in 1937 by $Y$. Milon, today home to the presidency of Rennes 1 University (photo M.-Y. Daire, 2020). 
sciences de Rennes (fig. 1), il rencontre Jean Cogné, avec qui il mène plusieurs fouilles archéologiques, et publie une vingtaine d'articles. Dès 1947, il prend simultanément les commandes du musée de Penmarc'h et de la $4^{\mathrm{e}}$ circonscription des Antiquités Préhistoriques du Secrétariat d'État aux Beaux-Arts qui dépend alors du ministère de l'Éducation nationale, qui couvre alors sept départements de l'Ouest; il restera directeur de son héritière, la circonscription de Bretagne, jusqu'en 1972.

En 1950, il soutient, à Rennes, sa thèse de doctorat ès sciences naturelles intitulée Armoricains et Bretons, étude anthropologique. Elle se veut un bilan provisoire de cinq années de recherche consacrées non seulement à l'anthropologie biologique, mais aussi préhistorique et ethnologique (Giot, 1951). Son objectif est d'éclairer, par la biologie des populations, certains aspects controversés des problèmes relatifs aux origines et à l'histoire de la population bretonne. Il tente d'évaluer, aux différentes périodes de la Protohistoire et de l'Histoire, les proportions relatives des diverses composantes humaines ayant contribué aux peuplements successifs de l'Armorique puis de la Bretagne.

Outre le fait qu'il s'intéresse à tous les sujets, sans exclusive, P.-R. Giot va cumuler, pendant plus de trois décennies, des responsabilités scientifiques et universitaires clés au niveau régional avec des mandats officiels du ministère de la Culture. Ce qui en fait le personnage central de l'archéologie de l'ouest de la France pendant toute cette période.

Instigateur du rattachement du musée de Penmarc'h à la faculté des sciences de l'université de Rennes, au cours des années 1950, P.-R. Giot crée le Laboratoire d'Anthropologie, à l'origine rattaché à l'Institut de géologie, laboratoire dont il assure la responsabilité jusqu’à son départ en retraite, en 1989. Dès les années 1950, il recrute alors des collaborateurs et équipe progressivement le laboratoire pour développer une recherche pluridisciplinaire : anthropologie biologique, géologie, pétrographie, chimie, etc. (Audouze, 2003; Collectif, 1990). Même si son travail est resté limité au contexte armoricain, il siège au conseil scientifique du Centre des Recherches Archéologiques tout comme les autres responsables des grands laboratoires de Préhistoire de l'époque : ceux d'André Leroi-Gourhan à la Sorbonne et au Collège de France, de François Bordes à Bordeaux et de Gabriel Camps à Aix-en-Provence (Audouze, 2003, note 28).

Pendant la seconde moitié du xx s., P.-R. Giot mettra peu à peu en place, à Rennes et à Nantes, des chercheurs qu'il forme et oriente vers telle ou telle spécialité et vers différentes instances: Jacques Briard (1933-2002) pour l'âge du Bronze (Collectif, 2002b), Jean L'Helgouac'h (1933-2000) (fig. 2 et 3) puis, à partir des années 1960, Roger Joussaume et Charles-Tanguy Le Roux (fig. 4) pour le Néolithique,
Jean-Laurent Monnier (fig. 5) pour la Préhistoire ancienne, puis Pierre Gouletquer qui travaillera successivement sur les briquetages protohistoriques puis le Mésolithique, dans la période comprise entre les années 1950 et $1980^{1}$. Ces chercheurs étaient épaulés par des ingénieurs et techniciens; ainsi, Jean-Roger Bourhis réalisait les analyses métallographiques, Yvan Onnée (fig. 3), Henri Poulain et Yannick Lecerf participaient aux campagnes de terrain et assuraient les relevés de terrain et dessins de mobiliers.

De la fin des années 1950 aux années 1990, P.-R. Giot cumulera ainsi, successivement voire simultanément, des responsabilités administratives et scientifiques à travers les fonctions de directeur de la quatrième circonscription des Antiquités préhistoriques, de conservateur en chef du musée de Carnac et plus durablement de celui de Penmarc'h, de chercheur au CNRS, de directeur du Laboratoire d'Anthropologie. Ce dernier évoluera ensuite en équipe de recherche dont nous retraçons infra l'historique.

\section{AUX ORIGINES : UN RÉSEAU D'ÉRUDITS LOCAUX TRAVERSÉ DE FRACTURES POLITIQUES}

Le CReAAH hérite d'une longue tradition régionale et interrégionale en matière de recherches archéologiques que reflète pour partie la dissémination territoriale actuelle de ses membres. Le quart nord-ouest de la France est en effet, après 1800, le berceau de multiples associations érudites dont les activités se centrent sur l'archéologie et sur l'histoire.

Caen est le foyer du mouvement initié par Arcisse de Caumont (1801-1873) à partir de 1820, avec la création de la Société des Antiquaires de Normandie (1824), puis de la Société Archéologique de France pour la conservation et la description des monuments historiques (1834), qui entreprend de structurer et de coordonner un réseau d'érudits revendiquant son autonomie face aux pouvoirs politiques centralisateurs parisiens (Gerson, 2003); celle-ci organise des Congrès archéologiques de France annuels (Bercé, 1986; Juhel, 2004). Selon le même agenda politique et scientifique, l'Institut des Provinces est créé au Mans, en 1839, sous l'égide de Caumont et de l'érudit manceau Thomas Cauvin (1762-1846); il est destiné à réunir une élite savante provinciale capable de rivaliser avec les membres de l'Institut de France parisien. Poitiers est quant à elle le siège, dès 1834 , de la Société des Antiquaires de l'Ouest.

1. Les chercheurs mentionnés ont tous intégré le CNRS, en dehors de C.-T. Le Roux qui a rejoint le ministère de la Culture. Par la suite, l'unité intégrera plusieurs chercheurs du CNRS ainsi que de nombreux enseignants-chercheurs et des chercheurs relevant du ministère de la Culture, de l'Inrap, de collectivités territoriales, voir [http://creaah.cnrs.fr] et infra. 

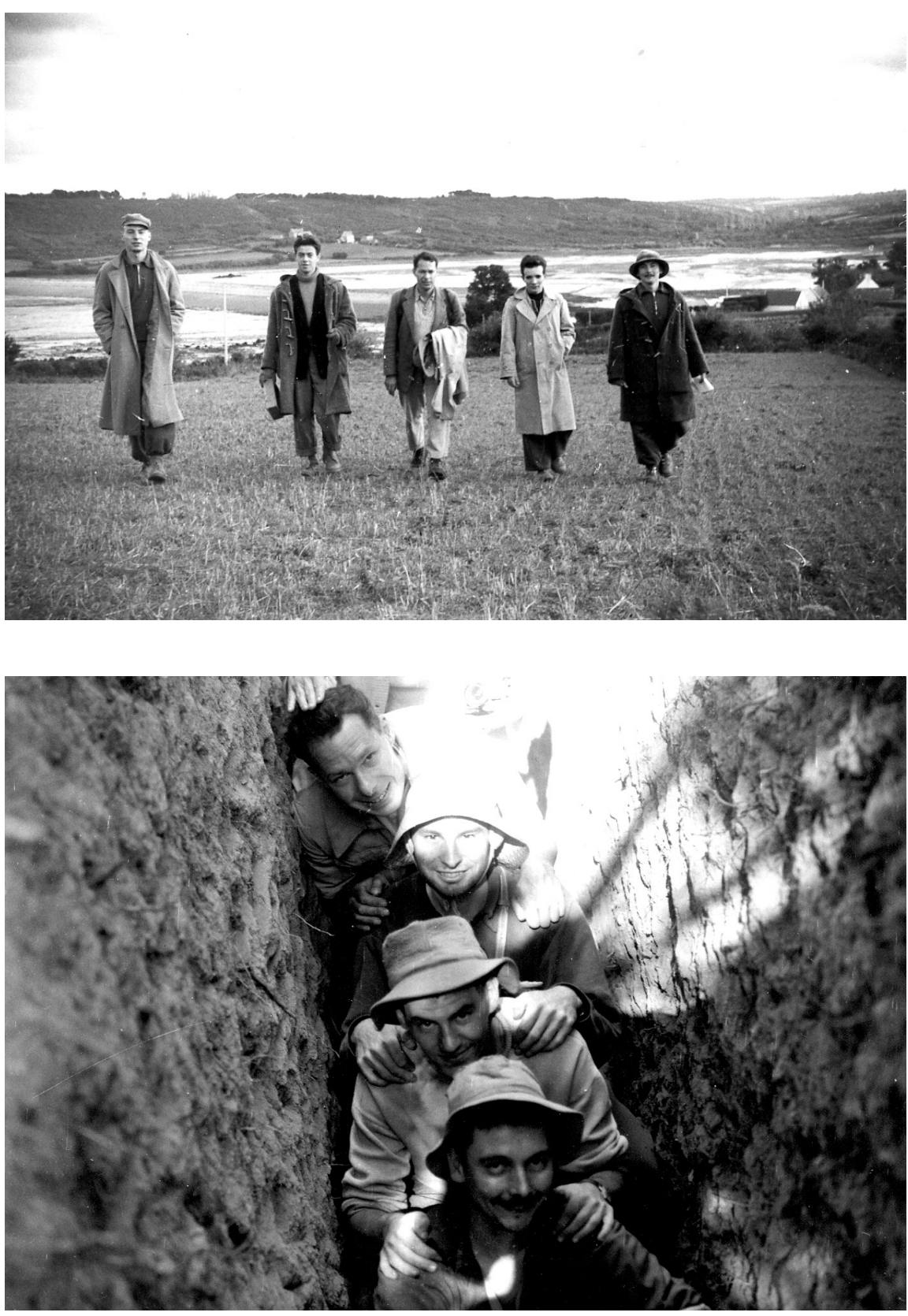

Figure 2 : Sur le site de Barnenez, de gauche à droite : Jean L'Helgouac'h, Yves Coppens, Pierre-Roland Giot, ?, et Jacques Briard (vers 1960?) (archives du Laboratoire Archéosciences-CReAAH).

Figure 2: Barnenez archaeological site, from left to right: Jean L'Helgouac'h, Yves Coppens, Pierre-Roland Giot, ?, and Jacques Briard (circa 1960?) (archives of the Archéosciences-CReAAH lab).
Figure 3 : Fouille du tumulus de Kerméné (1957-1958), de haut en bas : PierreRoland Giot, Jean L'Helgouac'h, Yvan Onnée et Jacques Briard (archives du Laboratoire Archéosciences-CReAAH). Figure 3: Excavation of the Kermené tumulus (1957-1858), from top to bottom: Pierre-Roland Giot, Jean L'Helgouac'h, Yvan Onnée and Jacques Briard (archives of the Archéosciences-CReAAH lab).
Si Nantes et Rennes ne sont pas au cœur de ce mouvement, elles sont les lieux d'une longue tradition érudite marquée par des figures d'antiquaires (Monteil, Santrot, 2011), dont le plus célèbre est, dans la première moitié du XVIII ${ }^{\mathrm{e}} \mathrm{s}$., Christophe-Paul de Robien (1698-1756), par ailleurs président à mortier au Parlement de Bretagne (Aubert, 2001). Après 1800, la Bretagne est, comme les autres régions, touchée par le mouvement des sociétés savantes (Chaline, 1995). La plupart consacrent une partie de leurs activités à l'archéologie, et certaines d'entre elles deviennent des acteurs majeurs du développement de la Préhistoire. Tel est le cas, tout particulièrement, de la Société polymathique du
Morbihan, fondée à Vannes en 1826 (Ars, 2002; Audren et al., 2005). Mettant en avant l'archéologie et la botanique dans son programme originel, elle se recentre rapidement sur la première et entreprend, dans les années 1850 et 1860 des fouilles importantes dans plusieurs tumuli, dont le tertre Saint-Michel de Carnac. Ces fouilles démontrent leur fonction funéraire et mettent à bas l'interprétation des dolmens comme autels sacrificiels qu'avaient privilégiée les celtomanes de l'époque romantique (Le Pennec, 2011).

Le monde breton des sociétés savantes est bouleversé, en 1843, par la création de l'Association bretonne. Rassemblant propriétaires terriens et érudits, centrée sur l'agronomie 


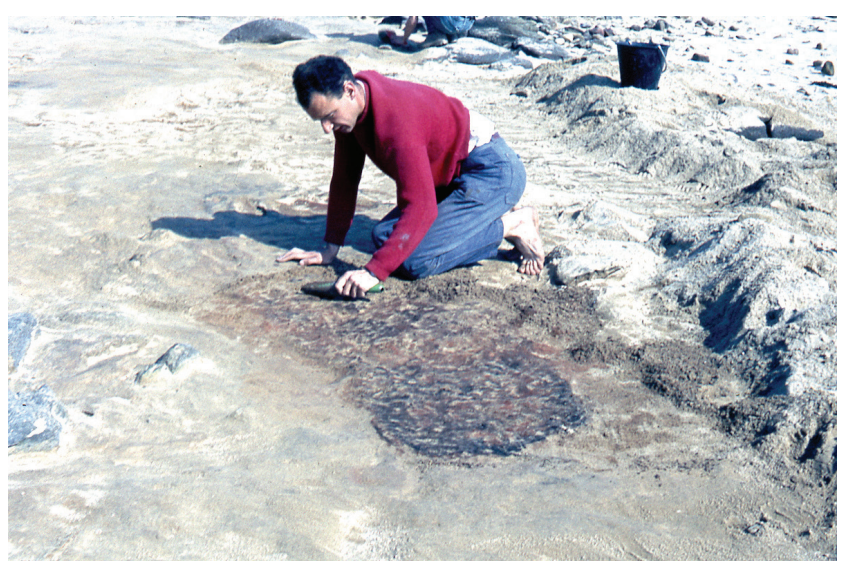

Figure 4 : Charles-Tanguy Le Roux sur la fouille du site de briquetages de Kerlavos à Trégastel en 1964 (dir. : P.-R. Giot) (archives du Laboratoire Archéosciences-CReAAH).

Figure 4: Charles-Tanguy Le Roux excavating on the Kerlavos 'briquetages' site (dir.: P.-R. Giot) (archives of the ArchéosciencesCReAAH laboratory).

mais aussi l'histoire et l'archéologie, cette nouvelle association d'échelle régionale a une dimension politique qui rappelle celle des réseaux structurés par Caumont. Elle est, comme la Société des Antiquaires de Normandie, dominée par une élite nobiliaire, catholique et conservatrice, qui associe convictions royalistes légitimistes et régionalisme, et qui lutte contre une centralisation parisienne perçue comme héritière du jacobinisme révolutionnaire ayant eu raison des anciennes libertés provinciales (Guiomar, 2019). Développant l'étude du passé local dans ses dimensions historiques, archéologiques et ethnographiques afin de retrouver - ou d'inventer - une identité régionale ancrée dans les traditions populaires et dans le catholicisme, promouvant le développement agricole, l'association se fait la porte-parole de mots d'ordre politiques d'opposition sous la monarchie de Juillet et sous le Second Empire, ce qui lui vaut une dissolution en 1859 avant sa renaissance en 1873 . Son activité, au sein des milieux érudits, les fractures entre «blancs» et "bleus ", héritage des conflits révolutionnaires, ces dissensions politiques expliquent, par exemple, la faiblesse des relations entre les membres de la Société polymathique du Morbihan, au sein de laquelle domine une prudente modération, et leurs homologues de la Société archéologique du Finistère fondée en 1845, plus nettement engagés dans l'Association bretonne. Armand du Chatellier (1797-1885), historien amateur, compte parmi les fondateurs de l'une comme de l'autre.

Traversé par ces tensions, le monde érudit breton n'en réalise pas moins durant le $\mathrm{XIX}^{\mathrm{e}} \mathrm{s}$. une entreprise importante d'inventaire, de fouilles et de préservation des vestiges archéologiques régionaux. Associations savantes et

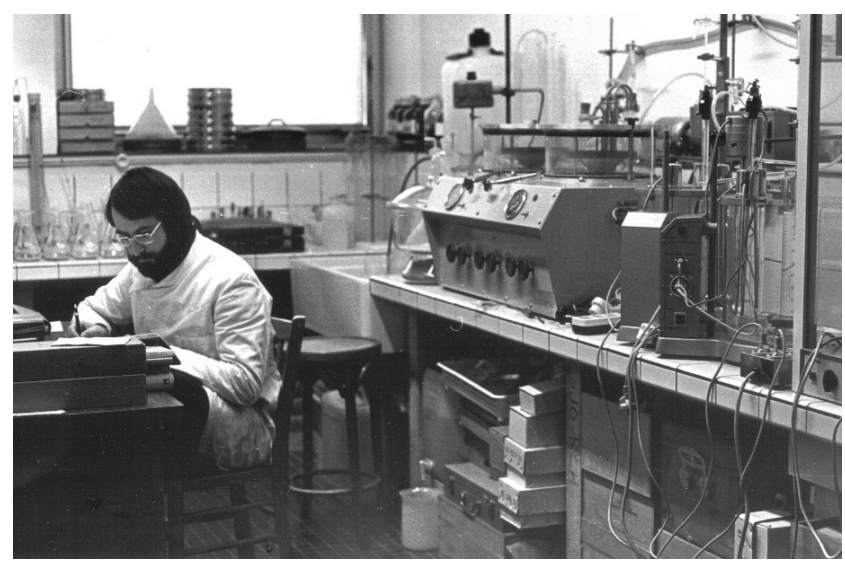

Figure 5 : Jean-Laurent Monnier dans le laboratoire de sédimentologie, à Beaulieu, en 1978 (cliché R. Monnier).

Figure 5: Jean-Laurent Monnier in the sedimentology lab, Beaulieu, in 1978 (photo R. Monnier).

autres amateurs sont à l'origine de nombreuses collections publiques et privées, dans lesquelles la Préhistoire occupe une place importante. Le musée archéologique de la Société polymathique du Morbihan, inauguré en 1853, offre l'une des plus notables (Le Pennec, 2011). À Carnac, le musée James-Miln est ouvert en 1882 (fig. 6). Il résulte du legs à la commune de la collection privée que le riche écossais Miln (1819-1881) avait réunie grâce à ses fouilles de sites gallo-romains et mégalithiques des environs dans les années 1870. Ce musée s'enrichit par la suite de dons et d'achats sous l'initiative de son gardien, puis conservateur, Zacharie Le Rouzic (1864-1939) (Riskine, 2000). Dans le Finistère, l'une des collections les plus réputées est

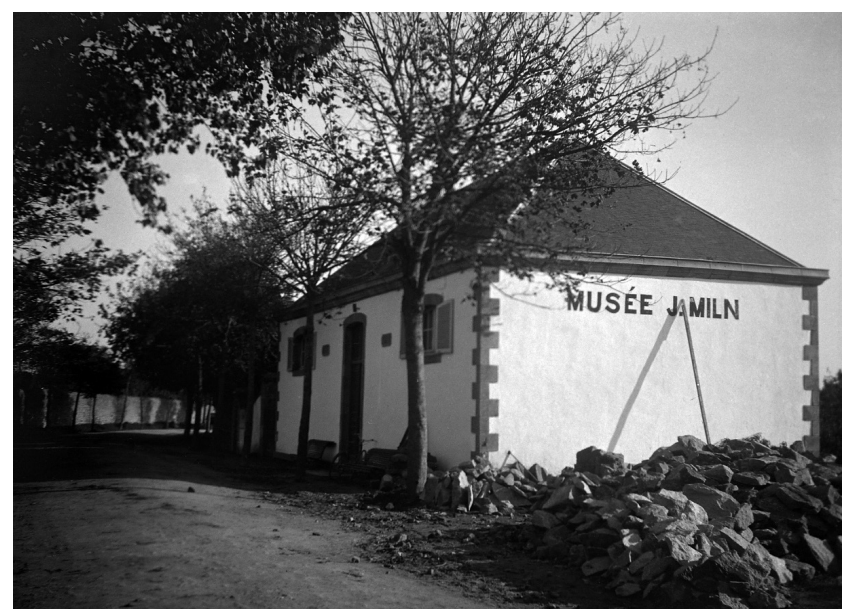

Figure 6 : Musée Miln-Le Rouzic, Carnac (cliché G. d'Ault du Mesnil, 1910, archives du Laboratoire Archéosciences-CReAAH). Figure 6: The Miln-Le Rouzic museum, Carnac (photo G. d'Ault du Mesnil, 1910, archives of the Archéosciences-CReAAH lab). 
celle que Paul du Chatellier (1833-1911), fils d'Armand, passionné d'archéologie et d'art, réunit au château familial de Kernuz grâce à ses fouilles et à la fortune familiale (Coativy, 2006). À côté de ces collections majeures, de nombreuses séries d'importance plus secondaire sont rassemblées à titre privé par des archéologues amateurs. Ceux-ci réunissent parfois des ensembles remarquables, tel le capitaine de frégate lorientais Louis Le Pontois (18381919) qui collecte près d'un millier de haches polies dans les deux dernières décennies du XIx ${ }^{e}$ s., avant de les céder en 1904 à du Chatellier (Langouët et al., 2007), ou encore le baron Maurice Halna du Fretay (1835-1901) dont la belle collection préhistorique est vendue aux enchères à l'hôtel Drouot en 1920 (Saint-Raymond, 2019). D'autres collections sont plus modestes, à l'instar de celle de Félix Gaillard (1832-1910), propriétaire de l'Hôtel du commerce à Plouharnel, qui rassemble dans son établissement, entre les années 1870 et 1880, un petit " musée ». Issu de ses fouilles et d'échanges, il est destiné tant à satisfaire sa passion pour l'archéologie qu'à attirer dans son hôtel les touristes qui profitent de l'arrivée du chemin de fer à partir de 1882 (Crowhurst, Gaillard, 2004). Beaucoup de ces collections sont dispersées après la mort de leur propriétaire et ont abouti, par fragments plus ou moins complets, dans les collections publiques, notamment au Musée d'archéologie nationale de Saint-Germain-en-Laye, acquéreur de la collection du Chatellier en 1924. Certaines viennent enrichir les collections publiques locales, tel le musée de Penmarc'h dont P.-R. Giot devient conservateur après la Seconde Guerre mondiale.

\section{Le musée de PenmarC'H}

La fonction de conservateur du musée de Penmarc'h occupera une grande place dans les activités scientifiques et la vie du chercheur qu'était P.-R. Giot et, à ce titre, il convient de s'attarder sur l'histoire de cet établissement et de la constitution de ses collections (fig. 7). L'exploitation et la valorisation du fonds d'archives relatif à ce musée, initiées depuis plusieurs années dans le cadre du projet Icare ${ }^{2}$, livrent des éléments de compréhension qui sont ici synthétisées (López-Romero, Daire, 2013; López-Romero, Le Gall, 2008; Daire, López-Romero, 2014; López-Romero, 2015).

La période 1850-1939 est marquée par un contexte international où la Préhistoire européenne trouve ses fondements en tant que discipline scientifique à part entière et par un cadre national où, on l'a vu, la vie scientifique tourne autour

2. Icare : Iconographie archéologique de l'ancien Laboratoire d'Anthropologie de Rennes, projet porté par M.-Y. Daire et E. López-Romero.

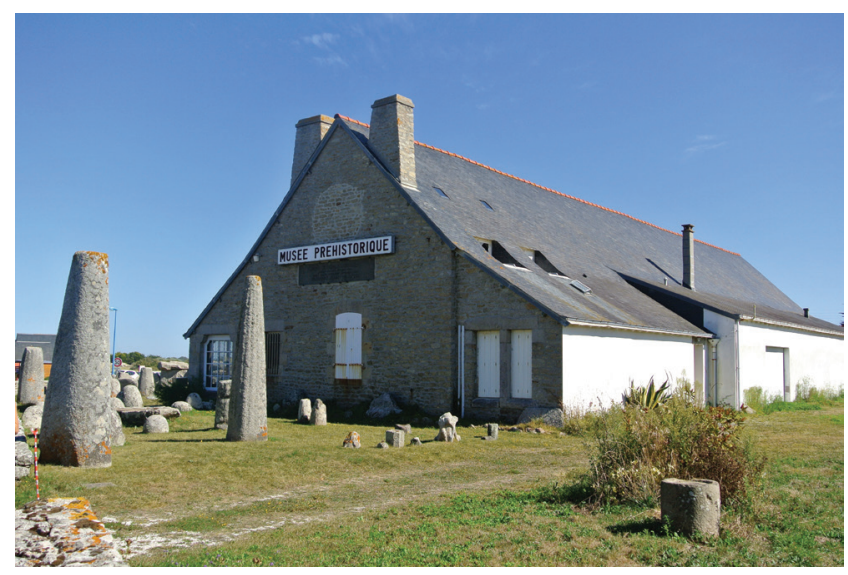

Figure 7 : Le musée préhistorique finistérien de Penmarc'h (cliché M.-Y. Daire, septembre 2012).

Figure 7: The Finistère Prehistory Museum in Penmarch (photo M.-Y. Daire, september 2012).

des sociétés savantes et des congrès scientifiques. Une prise de conscience sur le besoin de préservation des vestiges du passé apparait dès le premier tiers du XIX ${ }^{e}$ s. et se consolide lentement. Le nombre des revues scientifiques augmente considérablement et, à partir de 1887 et plus encore après 1913, une loi encadre " la conservation des monuments d'art ayant un intérêt historique et artistique " (Hurel, 2007, p. 96-108), tandis que les avancées dans le domaine de la photographie ouvrent aussi de nouvelles possibilités dans l'acquisition de ressources documentaires.

Dans le Finistère, plusieurs érudits (officiers de marine, médecins, hommes d'Église) passionnés d'archéologie fondent en 1921 la Société Civile du Musée d'Archéologie de Penmarc'h, qui prend l'année suivante le nom de Groupe finistérien d'études préhistoriques puis bénéficiera d'une reconnaissance nationale en tant qu'Institut Finistérien d'Études Préhistoriques (IFEP) dès 1926 (fig. 8) (López-Romero, Daire, 2013; Daire, López-Romero, 2014; Desailly, 1926; Motet et al., 1929; Monnier, 1992).

La création de la "Société » est basée sur la volonté de développer une méthodologie de fouille rigoureuse, reposant sur une organisation rationnelle et de stricts principes d'observation... Le groupe se compose, à l'origine, de Charles Bénard (1867-1931) - dit Bénard Le Pontois, président fondateur -, du commandant Alfred Devoir (1865-1923) et du peintre Georges Boisselier (1876-1943), très vite rejoints par l'abbé Favret (1875-1950), fondateur du musée d'Épernay en 1931, le chanoine Jean-Marie Abgrall (1846-1926), le docteur Mathurin Kermarec (1860-1934) et le commandant Émile Morel (1864-1927), entre autres. La rencontre entre Charles Bénard et l'abbé Favret s'était produite pendant la Première Guerre mondiale à l'hôpital militaire de Châlons- 
Figure 8 : En-tête de courrier de l'Institut Finistérien d'Études Préhistoriques (archives du Laboratoire Archéosciences-

Figure 8: Letterhead of the Institut Finistérien d'Etudes Préhistoriques (archives of the Archéosciences-CReAAH lab). CReAAH).

\section{INSTITUT FINISTÉRIEN D'ÉTUDES PRÉHISTORIQUES ftabllssement reconnu d'utilité publique par Décret du 12 Aout 1928 MUSÉ mational PRÉHistorioue de PENMARC'H}

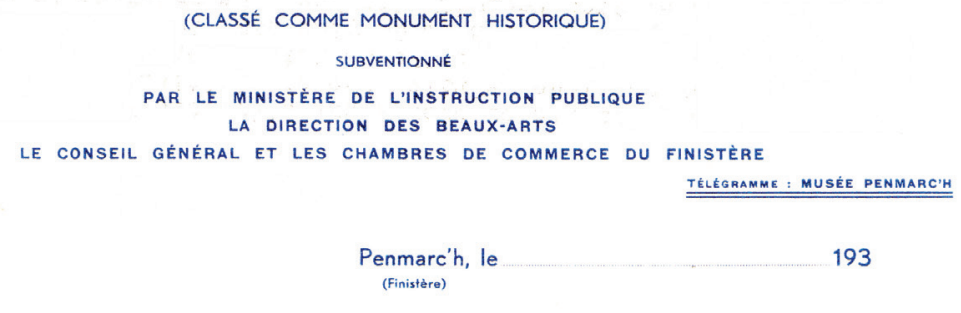

en-Champagne. La relation de Favret avec la Bretagne commence, quant à elle, en 1919, lorsqu'il participe en compagnie de Bénard aux fouilles sur le littoral et les îles du Finistère $^{3}$ (Daire et al., 2013). L'Institut Finistérien d'Études Préhistoriques bénéficie rapidement de l'adhésion d'illustres membres tels que Salomon Reinach (1858-1932), conservateur du musée de Saint-Germain-en-Laye ou encore du docteur Joseph-Louis Capitan (1854-1929).

Grâce à la mobilisation de fonds publics et privés, notamment ceux apportés par $\mathrm{M}^{\mathrm{me}}$ Bénard, née Phillipson, et le mécène Sir Robert Mond (magnat du nickel), la construction du "Musée Préhistorique " débute en 1922, immédiatement en arrière de la plage de Porz Carn à SaintGuénolé-Penmarc'h (Finistère), et la première salle est inaugurée en 1924. L’année suivante verra le classement des collections au titre des Monuments historiques tandis que la seconde salle ouvrira au public en 1927. Le musée abrite dès lors les collections d'objets et de documents accumulés par les fondateurs au fil de leurs recherches (Daire, LópezRomero, 2014).

Parmi les membres fondateurs, figure Alfred Devoir (1865-1926), que P.-R. Giot considérera comme la " tête pensante " du Groupe Finistérien d'Études Préhistoriques (Giot, 1984). Celui-ci s'est intéressé au mégalithisme des régions de Lorient, puis de Brest avant de se pencher sur la question de l'évolution de l'environnement côtier (Devoir, 1912, 1923). Il devient vice-président de la Société Archéologique du Finistère puis, entre 1902 et 1913, membre correspondant de la sous-commission des Monuments mégalithiques. Très concerné par le devenir des sites préhistoriques de Bretagne, il permet le classement de nombre d'entre eux au titre des Monuments historiques dont la tombe à couloir du Mougau à Commana (Finistère), monument qu'il achète en 1906 et fait classer en 1909 et

3. Communication personnelle de J.-R. Chatillon d'après un courrier de J.-J. Charpy, ancien directeur du musée archéologique d'Épernay. qui fut propriété de l'université de Rennes 1 pendant plusieurs décennies.

Par ses rapports avec Alfred Devoir et d'autres membres de l'IFEP, Geoffroy d'Ault du Mesnil (1842-1921) se présente également comme un acteur fondamental dans le creuset qui donnera lieu au « Musée Préhistorique ». De par sa formation originelle, liée à la géologie et la Préhistoire de la Bretagne sous les auspices du comte de Limur (1817-1901) et au sein de la Société Polymathique du Morbihan, mais aussi à cause de l'influence qu'il a exercée sur la recherche bretonne depuis les différents postes à responsabilité qu'il a occupés dans les instances parisiennes à une époque où la connaissance de la Préhistoire fait un grand bond (López-Romero et Le Gall, 2008; López-Romero, 2015), on peut affirmer que d'Ault du Mesnil a joué un rôle déterminant - bien que largement oublié - dans le développement de la Préhistoire régionale. En reconnaissance de ces contributions, et à titre posthume, une plaque faisant état d'une donation ${ }^{4}$ financière par son épouse lui a été dédiée au musée. On ne manquera pas non plus de signaler ici son rôle déterminant dans la formation initiale de jeunes chercheurs tels que Jacques de Morgan (1857-1924) (Jaunay, 1997, p. 97 et 255) ou Henri Breuil (1877-1961) (Hurel, 2011).

Parmi les autres personnalités liées à la Préhistoire finistérienne et à l'archéologie française de la fin du XIx ${ }^{e}$ et du début du Xx ${ }^{e}$ s., le controversé Charles Bénard (1867-1931) qui adopte indûment le nom de "Bénard-Le Pontois ${ }^{5}$ ", qu'il n'utilise cependant que dans le milieu archéologique, étudiera plusieurs nécropoles du Sud-Finistère (Saint-Urnel et Roz An Tremen à Plomeur), en alternance avec ses expéditions océanographiques. Ces études le conduiront à col-

4. Il s'agit probablement d'une donation d'archives, y compris les plaques et tirages photos qui font aujourd'hui partie des archives du laboratoire.

5. Après 1919, suite à la mort du commandant et archéologue amateur Louis Le Pontois (1838-1919), sans doute en témoignage d'admiration et même si aucun lien de parenté ne les unit. 
laborer avec l'abbé Favret ou encore G. Boisselier et, de manière plus anecdotique, à former un jeune étudiant du nom de... Théodore Monod (Daire et al., 2013). En 1929, Charles Bénard publie Le Finistère préhistorique (Bénard, 1929), ouvrage résultant de la collaboration entre plusieurs membres de l'IFEP, mais qui sera finalement édité sous sa seule signature et, cette même année, il succède au docteur Louis Capitan à la chaire de professeur d'Archéologie à l'École d'Anthropologie de Paris.

Le " Musée Préhistorique » de Penmarc’h eut pour premier conservateur, Charles Bénard, de 1922 jusqu’à 1931. Il fut remplacé dans cette fonction par Georges Boisselier, de 1932 à 1939. Le musée restera ensuite sans conservateur pendant la Seconde Guerre mondiale. La plage de Pors Carn, considérée comme propice à un éventuel débarquement, avait en effet été déclarée zone interdite par l'occupant jusqu’à ce que P.-R. Giot en prenne la direction et le rattache ainsi durablement à l'université de Rennes.

L'action de P.-R. Giot à Penmarc'h est révélatrice de la politique qu'il mène en tant que directeur de la circonscription des antiquités préhistoriques et directeur d'un laboratoire universitaire. Il s'efforce de restructurer la recherche et la gestion des collections en la centralisant sur un pôle académique unique, transformant les anciens musées locaux en stations scientifiques. Dans les mêmes années en effet, il négocie également, avec la commune et avec son conservateur, la cession du musée Miln-Le Rouzic de Carnac à l'Institut de géologie de Rennes. L'opération aboutit en 1948 et le projet d'un Institut de Préhistoire bretonne, installé dans de nouveaux locaux qui hébergeraient aussi le musée, est envisagé. Contrairement à Penmarc'h, ce rattachement ne dure que quelques années, le projet n'aboutit pas et le musée est rétrocédé à la commune en 1958. Cette politique de restructuration de la Préhistoire autour d'un pôle unique s'est accompagnée du transfert vers Rennes d'archives, de collections photographiques et parfois aussi de parties de collections des institutions rattachées (fig. 9 et 10). Elle explique l'exceptionnelle richesse du fonds documentaire ancien aujourd'hui conservé et valorisé au sein du Laboratoire Archéosciences-CReAAH de l'université de Rennes 1 (Daire, López-Romero, 2014), mais en rend également l'histoire très complexe.

\section{Des racines auX CREUSETS}

\section{Le creuset de la faculté des sciences de Rennes}

C'est dans l'immédiat après-guerre que sera créé le "Laboratoire d'Anthropologie " de la faculté des sciences, dénommé ensuite "Laboratoire d'Anthropolo-

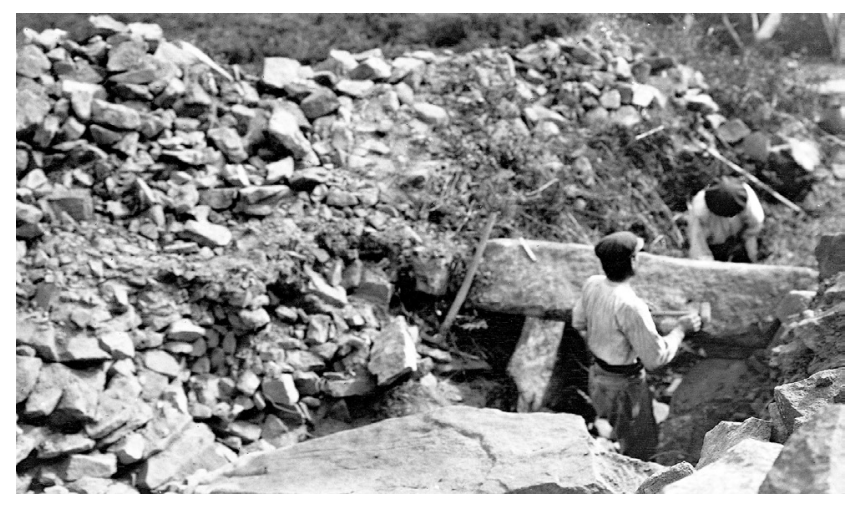

Figure 9 : Fouille du monument mégalithique de l'île Longue (Baden, Morbihan) (cliché G. d'Ault du Mesnil, 26 septembre 1907, archives du Laboratoire Archéosciences-CReAAH).

Figure 9: Excavation of the megalithic monument on Ille Longue (Baden, Morbihan) (photo G. d'Ault du Mesnil, September 26, 1907, archives of the Archéosciences-CReAAH lab).

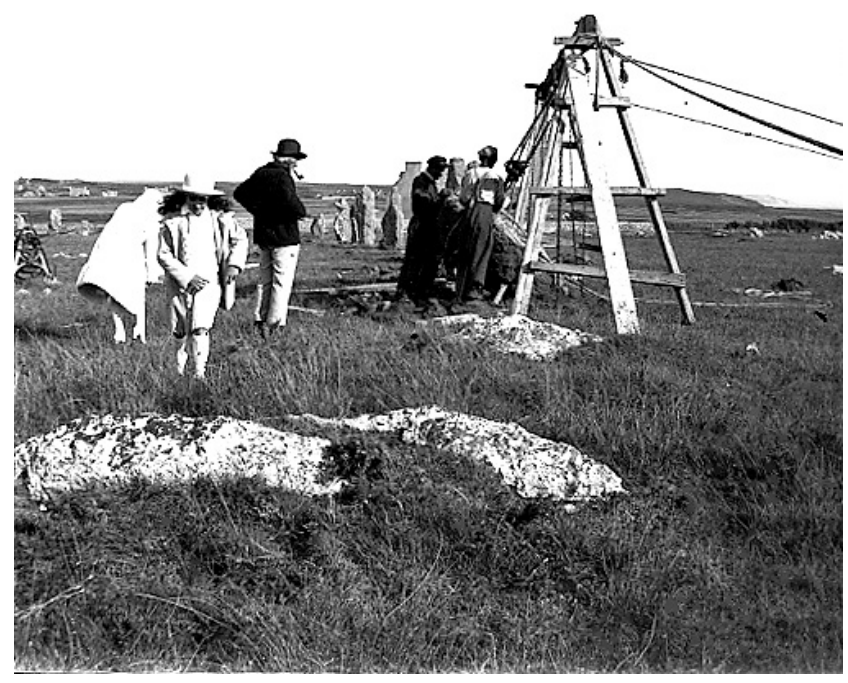

Figure 10 : Restauration des alignements de stèles de Lagatjar à Camaret (Finistère) en 1928-1929 (cliché C. Bénard, archives du Laboratoire Archéosciences-CReAAH).

Figure 10: Restoration of the stelae alignments of Lagatjar at Camaret (Finistère) in 1928-1929 (photo C. Bénard, archives of the Archéosciences-CReAAH laboratory).

gie Préhistorique " puis "Laboratoire d'Anthropologie, Préhistoire, Protohistoire et Quaternaire armoricains " de l'université de Rennes, puis l'Équipe de Recherche no $27 \mathrm{du}$ CNRS à la fin des années 1960. Des liens très forts unissaient P.-R. Giot et les membres du laboratoire à l'Institut de géologie de Rennes, au point que certains collègues, tels Yves 
Milon (1897-1987)6 ou Léon Collin (1872-1945), firent don d'une partie de leurs archives. Elles sont aujourd'hui encore conservées au Laboratoire Archéosciences. Le laboratoire connut un déménagement dans les années 1960, depuis les anciens locaux de l'Institut de géologie dans le centre de Rennes vers le campus scientifique de Beaulieu (fig. 11), ce qui préserva l'intégrité de ce fonds documentaire, qui constitue une source d'information de premier ordre pour la compréhension du développement de la Préhistoire et de l'archéologie en Bretagne et un riche potentiel scientifique (López-Romero, Daire, 2013; Daire, López-Romero, 2014).

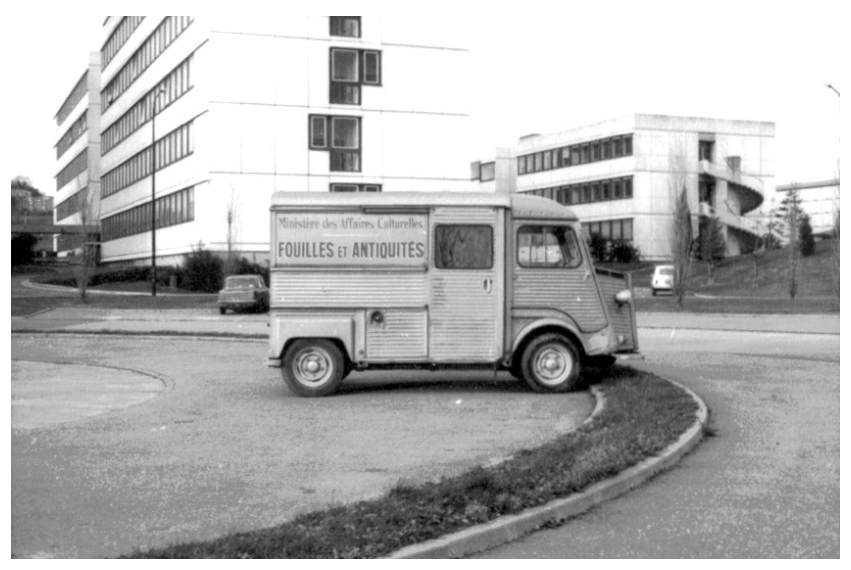

Figure 11 : Le camion des "Fouilles et Antiquités " sur le campus de Beaulieu à Rennes, vers 1960 (archives du Laboratoire Archéosciences-CReAAH).

Figure 11: The "excavations and antiquities" truck on the Beaulieu campus, around 1960 (archives of the Archéosciences-CReAAH lab).

Sur le site rennais du campus de Beaulieu, les années 1980 virent la naissance du Laboratoire d'Archéométrie, fondé par Loïc Langouët (1941-2018) et Louis Goulpeau (1939-2019), enseignants-chercheurs en physique et électronique de l'université Rennes 1, qui développaient alors l'unité de datation par archéomagnétisme - avec une première sur matériaux déplacés -, parallèlement aux activités de prospections géophysiques et de thermoluminescence (fig. 12) (Collectif, 2018). Avec A. Hesse et A. Tabbagh, L. Langouët fondera en 1976 le GMPCA (Groupe des Méthodes Physiques et Chimiques appliquées à l'Archéologie), qui deviendra en 1987 le Groupe des Méthodes Pluridisciplinaires Contribuant à l'Archéologie, afin d'intégrer les sciences de la nature et de la vie, ainsi que la Revue d'Archéométrie, devenue depuis la revue Archéosciences-Revue d'Archéométrie. Ces fondateurs constataient en effet, dans

6. Y. Milon fut d'ailleurs le premier directeur de la quatrième circonscription jusque vers 1955, avec P.-R. Giot comme adjoint. leur déclaration d'intention, que, pour " de nombreux archéologues, les recherches de terrain et de laboratoire sur les milieux et les matériels, objets de leur étude, ne se ramenaient plus à une simple description et analyse érudites, mais qu'un certain nombre de progrès dans le sens de la précision et de la rigueur exigeaient l'introduction de moyens nouveaux généralement issus des disciplines dites exactes ». Il en résultait que "l'évolution [de l'archéologie] considérée sous son jour le plus général, consacr[ait] en fait l'apparition de ce qu'il est convenu d'appeler la pluridisciplinarité. Celle-ci ne [pouvait] être fructueuse que dans le cadre d'une coopération intime et profonde des deux parties engagées dans le dialogue, chacune, en définitive, faisant siens problèmes et techniques de l'autre " (source : https://gmpca.fr/historiquede-l-association).

À la fin des années 1980, le déménagement du laboratoire permit un rapprochement physique avec le Laboratoire d'Anthropologie, ce qui s'accompagna d'une fusion institutionnelle d'où naquit, à l'initiative du CNRS, une première structure dénommée Unité Propre de Recherche (UPR 403) en 1988. Le rapprochement physique des personnels du CNRS (chercheurs et ingénieurs) de l'UPR 403 et des enseignants-chercheurs du Laboratoire d'Archéométrie, dans les actuels bâtiments 24 et 25 du campus de Beaulieu, posa les bases structurelles du futur laboratoire (fig. 13). Cette fusion explique également que, du point de vue universitaire, après avoir longtemps été rattaché à l'Institut de géologie, le laboratoire rennais se soit ensuite rapproché de la Physique (département Structure et Propriété de la Matière, SPM) de l'université Rennes 1, dont il a relevé jusque dans les années 2010.

\section{Le creuset de la faculté des lettres de Rennes}

Parallèlement, la faculté des lettres de Rennes aujourd'hui université Rennes 2 - vit la création, à la fin des années 1950, du "Laboratoire d'Archéologie " par la suite baptisé du nom de son créateur, Pierre Merlat (19111959) (fig. 14), professeur et doyen de la faculté des lettres et Sciences humaines de Rennes (1948-1959). Historien, archéologue et figure illustre de la guerre et de la Résistance, il a enseigné l'histoire et l'archéologie du monde romain dans cet établissement (Pape et al., 1960). À l'échelle régionale, ce laboratoire dirigé ensuite par Louis Pape (19332014), développa de nombreux programmes de terrain sur plusieurs sites d'époques historiques, dont notamment les fouilles pluriannuelles de l'agglomération secondaire antique de Kerilien à Plounéventer (Finistère), dirigées par Louis Pape (Aubin et Provost 2015), ou encore celles de l'abbaye de Landévennec (Finistère) dirigées pendant près de vingtcinq années par Annie Bardel. 

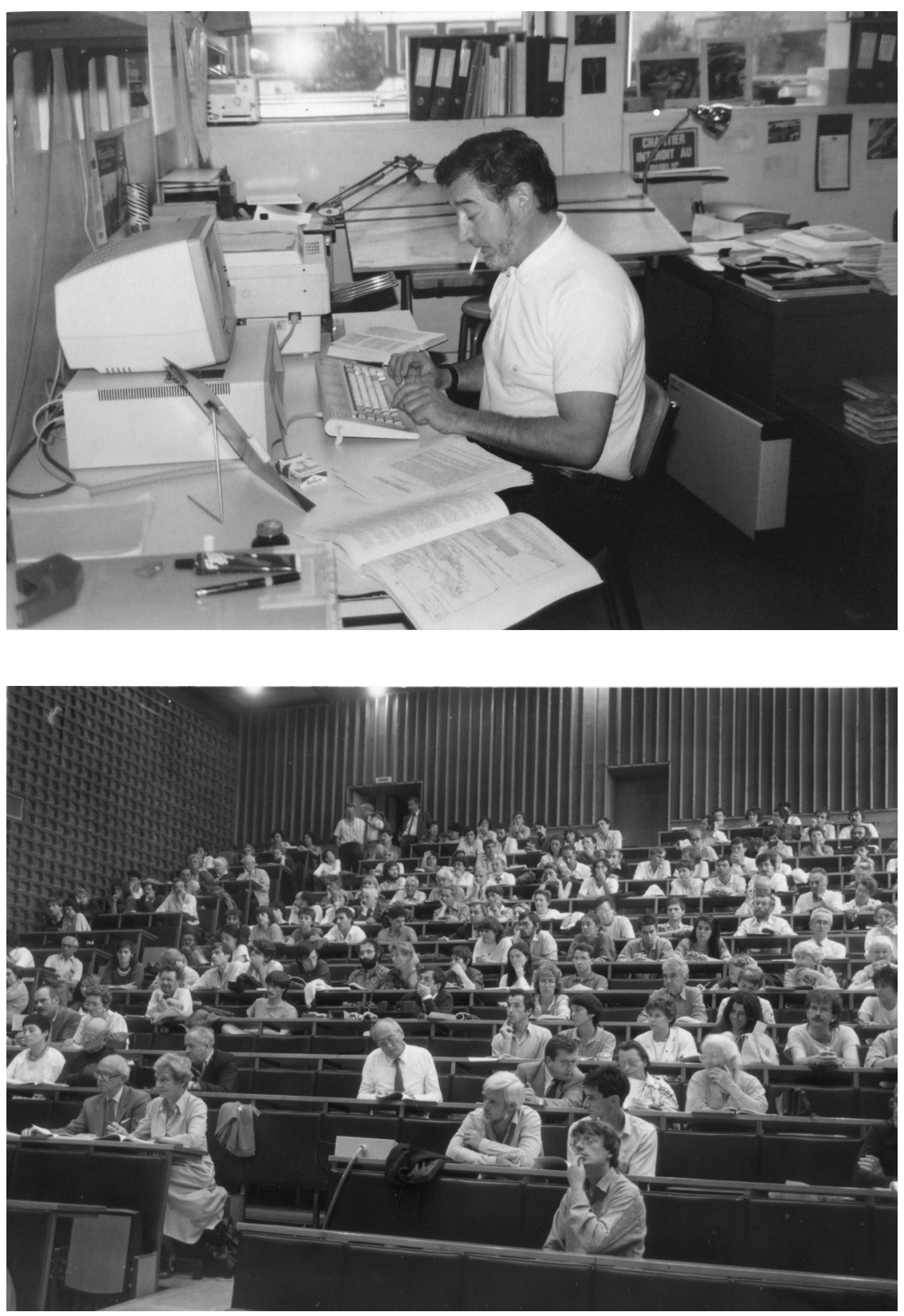

Figure 12 : Loïc Langouët au Laboratoire d'Archéométrie, à Beaulieu, vers 1980 (archives privées).

Figure 12: Loïc Langouët in the Archaeometry laboratory, Beaulieu, circa 1980 (private archives).

Figure 13 : La " Journée » de préhistoire et d'archéologie organisée annuellement par l'unité depuis plus de trente ans, ici fin des années 1970. On reconnaît aux deux premiers rangs : D. Marguerie, G. Querré, M. Le Goffic, J. Peuziat, F. et F. Quérat... et, debout en haut de l'amphi, L. Langouët (archives du Laboratoire Archéosciences, CReAAH).

Figure 13: The prehistory and archeology "Day", organized annually by the team for more than 30 years, here at the end of the 1970s. We recognize in the first two rows: D. Marguerie, G. Querré, M. Le Goffic, J. Peuziat, F. and F. Quérat... and, standing at the top of the amphitheatre, L. Langouet (archives of the Archeosciences lab, CReAAH).
Le "Laboratoire d'Archéologie et d'Histoire Merlat " (LAHM), ainsi rebaptisé en 2009, réunit dans un seul organisme de recherche les anciens laboratoires de l'université Rennes 2 "Pierre Merlat », "CERAMA » (Archéologie), et "CRESCAM» (Histoire antique). Le nom de cette nouvelle institution (Laboratoire d'Archéologie et d'Histoire Merlat, LAHM) souligne avant tout la vocation pluridisciplinaire du laboratoire (en archéologie et en histoire) qui s'inscrit dans la tradition d'un ancien laboratoire rennais initialement dédié à l'archéologie et à l'histoire des périodes historiques dans l'ouest de la France, avant de connaître une large ouverture nationale et internationale.

\section{Le creuset nantais}

On doit à Jean-René Jannot (1936-), archéologue et professeur à l'université de Nantes, le développement du département d'histoire de l'art et d'archéologie durant la période où il dirigea l'UFR d'Histoire et de Sociologie. La composante historique nantaise du CReAAH trouve ses origines dans le Laboratoire de Préhistoire Armoricaine de l'université de Nantes, crée en 1989 par J. L'Helgouac'h qui en assurera la direction jusqu'à son départ en retraite (Guyodo et al., 2019) (fig. 3 et 4). Après avoir réalisé à Rennes ses études puis sa thèse sur Les sépultures mégalithiques en Armorique, 
sous la direction de P.-R. Giot, et participé à de nombreux chantiers de fouilles en Bretagne, J. L'Helgouac'h, devenu directeur de recherche, prend pendant vingt ans - entre 1969 et 1989 - la direction de la circonscription des antiquités préhistoriques des Pays de la Loire et dirige de nombreux chantiers sur des sites mégalithiques dans tout l'ouest de la France (Champ-Grosset à Quessoy, Crec'h Quillié à Saint-Quay-Perros, Prajou-Menhir à Trébeurden, Gâvres, Larcuste à Colpo, Dissignac, Pornic, Locmariaquer, etc.). À cette occasion, il démontre des phénomènes de réemploi et de probable iconoclasie dans la grande architecture mégalithique armoricaine, à travers une retentissante publication Les idoles qu'on abat... (L'Helgouac'h, 1983). Parallèlement, il sera co-fondateur de la Revue Archéologique de l'Ouest en 1984, membre du Conseil supérieur de la recherche archéologique (1979-1984), de la Commission supérieure des Monuments historiques (1982-1985) et du conseil scientifique du Centre national de Préhistoire.

Le laboratoire nantais fut rejoint par Roger Joussaume (1938-), chercheur au CNRS, dont les recherches portaient sur le mégalithisme et le Néolithique de la France du CentreOuest, entre Loire et Gironde, par la fouille de nombreux dolmens jusqu'en Limousin et celle de la grande enceinte néolithique de Champ-Durand à Nieul-sur-l'Autise, en Vendée. Hors de France, il a travaillé sur le mégalithisme et l'ethnoarchéologie à Madagascar, en Indonésie et plus spécialement dans la Corne de l'Afrique : en Éthiopie, à Djibouti et en Somalie, trois pays où il a également développé une recherche spécifique sur l'art rupestre préhistorique.

En 1991, le Laboratoire de Préhistoire fut rejoint par un chercheur du CNRS, Serge Cassen, qui en prend la direction. Ce laboratoire devient ensuite le Laboratoire de recherche Archéologie et Architecture, à l'issue de la fusion avec le Laboratoire de Paléoenvironnement de la faculté des sciences de Nantes créé par Lionel Visset, enseignant-chercheur; cette composante intégrera ensuite l'UMR nouvellement créée.

\section{Naissance d'une Unité Mixte DE RECHERCHE (UMR)}

Les années 1990 virent à l'échelle nationale la création des Unités Mixtes de Recherche (UMR) et la disparition progressive des Unités Propres de Recherche (UPR), dans une volonté du CNRS et des acteurs de la recherche nationale de développer de nouveaux types de contrats de partenariat. À l'échelle nationale, la transformation des Unités Propres en Unité Mixtes vit quelques unités de recherche françaises intégrer des agents du ministère de la Culture qui devint alors aussi tutelle des laboratoires.

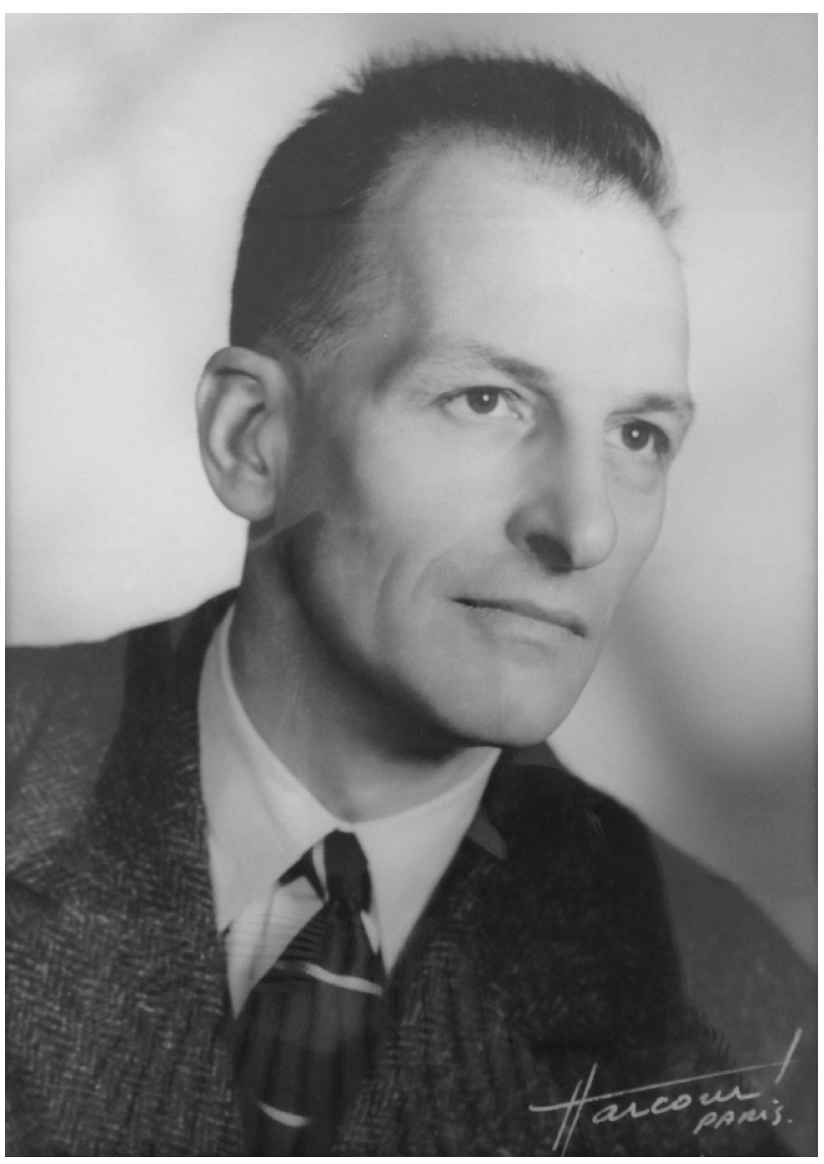

Figure 14 : Pierre Merlat, fondateur du Laboratoire d'Archéologie de la faculté des lettres de Rennes (cliché Harcourt).

Figure 14: Pierre Merlat, founder of the archeology laboratory of the Faculty of Letters of Rennes (photo Harcourt).

Le Laboratoire d'Anthropologie de l'immédiat aprèsguerre avait donné naissance, à la fin des années 1960, à une équipe de recherche labellisée par le CNRS, l'ER 27, qui se transformera en Unité Propre de Recherche (UPR 403) en 1988, par l'association des Laboratoires d'Anthropologie et d'Archéométrie de l'université de Rennes. Puis J.-L. Monnier, dernier directeur de l'UPR 403, fut sollicité et appuyé par la direction du CNRS pour créer une grande Unité Mixte de Recherche (UMR) dans l'ouest de la France, centrée à Rennes, au laboratoire de l'université Rennes 1, le but étant de mettre des moyens renforcés en commun et de favoriser les meilleures relations et collaborations possibles entre les différents partenaires, notamment avec le ministère de la Culture et ses services régionaux de l'Archéologie (SRA) au sein des Directions Régionales des Affaires Culturelles (DRAC). Ainsi naquit, en 1991, l'Unité Mixte de Recherche, alors rejointe formellement par le Laboratoire d'Archéologie et d'Histoire « Pierre Merlat » de l'université Rennes 2. 
Dès lors, le laboratoire, devenu UMR 153 puis UMR 6566 $\mathrm{C} 2 \mathrm{~A}$ "Civilisations atlantiques et Archéosciences ", intégra des personnels du CNRS ${ }^{7}$, des universités Rennes 1 et Rennes 2, du ministère de la Culture, puis de l'université de Nantes et plus récemment de l'Inrap et de l'université du Mans. En 2008, l'UMR C2A est rebaptisé CReAAH "Centre de Recherche en Archéologie, Archéosciences, Histoire ", afin de spécifier le tournant marqué par l'arrivée de nombreux collègues historiens des universités de Rennes 2 (en 2008) puis du Mans (en 2010) dont les territoires de recherche ne se limitent pas aux régions atlantiques. Les évolutions les plus récentes de la recherche archéologique française, notamment le développement de l'archéologie préventive depuis les années 1980 (création de l'Afan puis de l'Inrap), puis l'ouverture à la privatisation de l'archéologie, ont redistribué les cartes, tant du point de vue scientifique (volumes et modalités d'acquisition des données primaires, méthodes) que du point de vue de l'approche socio-historique de la discipline. Attractive, l'unité attire de plus en plus de membres travaillant dans le champ de l'archéologie préventive.

La constitution d'un laboratoire de recherche répond à des mécanismes structurels (voire politiques) tels qu'ils sont résumés supra, mais repose également sur des logiques scientifiques et l'évolution des disciplines. Sur ce plan, le CReAAH se situe à la fois dans la continuité de certaines thématiques de recherche et dans l'évolution des méthodes et des approches. Ici, il faut évoquer l'historique des compétences en Préhistoire et Protohistoire (Préhistoire ancienne ou encore mégalithisme) et en archéométrie, mais aussi l'élargissement des champs chronologiques (avec l'ouverture actuelle aux périodes historiques) et disciplinaires comme les archéosciences; au sein du laboratoire, ces dernières se sont progressivement désengagées des méthodes de datations physico-chimiques pour développer des compétences en matière de paléoenvironnements, tout en maintenant un haut niveau d'expertise dans l'étude des matériaux (inorganiques et organiques). Fin 2019, cette Unité Mixte de Recherche comptait 123 chercheurs, ingénieurs et techniciens ainsi que 45 doctorants, investis dans les programmes de recherche nationaux et internationaux des équipes qui la composent, ainsi que dans plusieurs formations universitaires, notamment au niveau des masters portés par le CReAAH à Rennes, Nantes et en Amérique du Sud ${ }^{8}$.

La suite de l'histoire reste à écrire...

7. En 1989, Marie-Yvane Daire et Philippe Lanos furent recrutés comme chercheurs par le CNRS et affectés au site rennais. Ils furent par la suite rejoints par d'autres chercheurs du CNRS tels que Jean-Pierre Pautreau, José Gomez de Soto, Luc Laporte... Voir la liste des membres sur le site web de l'unité.

8. [https://creaah.cnrs.fr/].

\section{Bibliographie}

Ars F., 2002 - La Société polymathique du Morbihan et la conservation du patrimoine mégalithique, 1826-1939, thèse de doctorat d'histoire, université de Nantes.

Aubert G., 2001 - Le Président de Robien, gentilhomme et savant dans la Bretagne des Lumières, Rennes, Presses universitaires de Rennes, 396 p.

Aubin G., Provost G., 2015, «In memoriam. Louis Pape (19332014)", Revue archéologique de l'Ouest, 32, p. 9-12.

Audouze F., 2003 - "La préhistoire et le CNRS », La revue pour l'histoire du CNRS, 8, 17 p.

Audren C., Frélaux B., Le Saux M.-F. (dir.), 2005 - La Société polymathique du Morbihan, Vannes, musée de Vannes.

BÉnARd C., 1929 - Le Finistère préhistorique, Paris, Publications de l'Institut International d'Anthropologie 3, Librairie Émile Noury, 337 p.

Bercé F., 1986 - "Arcisse de Caumont et les sociétés savantes ", dans Nora P. (dir.), Les lieux de mémoire, t. 2 : La Nation, Paris, Gallimard, p. 533-567.

Chaline J.-P., 1995 - Sociabilité et érudition. Les sociétés savantes en France, $X I X^{e}-X X^{e}$ s., Paris, CTHS, 270 p.

Coativy Y. (dir.) - 2006, Paul du Chatellier, collectionneur finistérien (1833-1911), Brest, UBO.

Collectif, 1990 - «Pierre-Roland Giot. Dans La Bretagne et l'Europe préhistoriques. Mémoire en hommage à Pierre-Roland Giot ", Revue archéologique de l'Ouest, suppl. 2, p. 5-9.

Collectif, 2002a - "Le Comité de Rédaction. In memoriam : Pierre-Roland Giot ", Revue archéologique de l'Ouest, 19, p. 5-6.

Collectif, 2002b - «Le Comité de Rédaction. In memoriam: Jacques Briard ", Revue archéologique de l'Ouest, 19, p. 6.

Collectif, 2018 - "Loïc Langouët, hommage ", Revue archéologique de l'Ouest, 35, p. 7-22.

Crowhurst H., Gaillard P., 2004 - Mémoire de pierres à Plouharnel. Photographies et textes inédits, 1870 à 1895, de Félix Gaillard, Plouharnel, HCom éditions, 81 p.

Daire M.-Y., López-Romero E., 2014 - "Écrire une page de l'histoire de l'archéologie : la collection documentaire de l'ancien Laboratoire d'Anthropologie de Rennes ", Antiquités Nationales, 44, p. 145-156.

Daire M.-Y., López-Romero E., Le Gall C., 2013 - «Théodore Monod (1902-2000) et l'archéologie bretonne : note sur un épisode méconnu de la vie du fou du désert ", Revue archéologique de l'Ouest, 30, p. 289-301.

Desailly M., 1926 - "Séance du 23 décembre 1926 ", Bulletin de la Société préhistorique française, 23, 12, p. 300.

Devorr A., 1912 - "Témoins mégalithiques des variations des lignes des rivages armoricains ", Bulletin de la Société archéologique du Finistère, 39, p. 220-239.

Devorr A., 1923 - «Un épisode de la submersion des rivages bretons : le pont Crac'h et les vestiges préhistoriques aux abords 
du Ras Aber Wrac'h », Bulletin de la Société géographique et minéralogique de Bretagne, 4, p. 150-176.

Gerson S., 2003 - The Pride of Place, Local Memories and Political Culture in Nineteenth-Century France, Ithaca, Londres, Cornell University Press.

Giot P.-R, 1951 - Armoricains et bretons, étude anthropologique, Rennes, travaux du Laboratoire d'Anthropologie de la faculté des sciences de Rennes, Mémoires de la Société géologique et minière de Bretagne, 10 (publication d'une thèse de sciences, université de Rennes).

Giот P.-R, 1984 - «Un préhistorien méconnu : Alfred Devoir ", Bulletin de la Société archéologique du Finistère, 113, p. 10-12.

Guiomar J.-Y., 2019 [1987] - Le Bretonisme. Les historiens bretons au XIX s., Rennes, Presses universitaires de Rennes.

Guyodo J.-N., Dieulefet G., Levéziel É., Mouchard J., 2019 - 1989-2019: 30 ans de recherches au LARA. Plongée dans les archives du Laboratoire de Recherche Archéologie et Architectures, catalogue d'exposition, Nantes, université de Nantes.

Hurel A., 2007 - La France préhistorienne de 1789 à 1941, Paris, CNRS Éditions.

Hurel A., 2011 - L'abbé Breuil. Un préhistorien dans le siècle, Paris, CNRS Éditions.

Jaunay A., 1997 - Mémoires de Jacques de Morgan, 1857-1924. Souvenirs d'un archéologue, Paris, L'Harmattan.

Juhel V. (dir.), 2004 - Arcisse de Caumont (1801-1873), érudit normand et fondateur de l'archéologie française, actes du colloque international de Caen (14-16 juin 2001), Caen, Société des Antiquaires de Normandie, 514 p. (Mémoires de la Société des Antiquaires de Normandie, 40).

Langouët L., Bihan S., Gouézin P., López-Romero E., 2007 - "Louis Le Pontois et les monuments mégalithiques de l'île de Groix ", Bulletin de l'Association Manche Atlantique pour la Recherche Archéologique dans les Îles (AMARAI), 20, p. 5-31.

L'Helgouac'H J., 1983 - "Les idoles qu'on abat... ", Bulletin de la Société polymathique du Morbihan, 110, p. 57-68.

Le Pennec C., 2011 - «La Société polymathique et la naissance de collections archéologiques en Morbihan ", Annales de Bretagne et des Pays de l'Ouest, 118, 3, p. 73-96.
López-Romero E., 2015 - "Geology meets Archaeology. Rediscovering the Life and Work of Geoffroy d'Ault du Mesnil (1842-1921) ", Proceedings of the Geologists Association, 126, 1, p. 143-153.

López-Romero E., Daire M.-Y., 2013 - « The ICARE Project: Insights into the Formation and Consolidation of Archaeology in Western France (ca. 1850-1990) ", Bulletin of the History of Archaeology, 23, 1, 3, p. 1-11.

López-Romero E., Le Gall C., 2008 - « Le fonds documentaire de Geoffroy d'Ault du Mesnil (1842-1921) et la recherche préhistorique en France ", Les Dossiers du centre régional d'Archéologie d'Alet, 36, p. 65-72.

Monnier J.-L., 1992 - « Le Musée de Préhistoire finistérienne à Penmarch, au carrefour des sciences. De la recherche à la valorisation culturelle et touristique du patrimoine archéologique ", Le Pays Bigouden à la croisée des chemins, actes du colloque de Pont-l'Abbé, novembre 1992, Pont-l'Abbé, Cap-Caval, Association de promotion du Pays Bigouden-Cap Sizun, université de Bretagne occidentale, p. 51-62.

Monteil M., SAntrot J. (dir.), 2011 - « La naissance de l'archéologie régionale dans l'Ouest armoricain ", Annales de Bretagne et des Pays de l'Ouest, 118-3, p. 9-32.

Motet C., Capitan L., Bénard C., 1929 - « Le musée préhistorique de Penmarc'h ", L'Illustration économique et financière, supplément au numéro du 9 mars, p. 67-72.

Pape L., Meynier A., Sauzin L., Henry P., Marache R., 1960, «Discours prononcé aux obsèques de M. Pierre Merlat, doyen de la Faculté des Lettres et Sciences humaines de Rennes, le 19 octobre 1959 ", Annales de Bretagne, 67-1, p. 4-32.

Riskine A.-E., 2000 - Musée de Préhistoire, James-Miln, Zacharie Le Rouzic : 450000 ans d'histoire au pays de Carnac, Carnac, musée de Préhistoire.

SAInT-Raymond L., 2019 - «Entre spécimen et belle série : la paradoxale mise en art des objets préhistoriques en vente publique (1882-1941) ", Revue de l'art, 206, p. 11-18. 